\title{
Long-term memory shapes the primary olfactory center of an insect brain
}

\author{
Benoît Hourcade, ${ }^{1,2}$ Emmanuel Perisse, ${ }^{1,2}$ Jean-Marc Devaud, ${ }^{1}$ \\ and Jean-Christophe Sandoz ${ }^{1,3}$ \\ ${ }^{1}$ Research Centre on Animal Cognition, CNRS, University Paul-Sabatier (UMR 5169), 31062 Toulouse cedex 04, France
}

\begin{abstract}
The storage of stable memories is generally considered to rely on changes in the functional properties and/or the synaptic connectivity of neural networks. However, these changes are not easily tractable given the complexity of the learning procedures and brain circuits studied. Such a search can be narrowed down by studying memories of specific stimuli in a given sensory modality and by working on networks with a modular and relatively simple organization. We have therefore focused on associative memories of individual odors and the possible related changes in the honeybee primary olfactory center, the antennal lobe (AL). As this brain structure is organized in well-identified morpho-functional units, the glomeruli, we looked for evidence of structural and functional plasticity in these units in relation with the bees' ability to store long-term memories (LTMs) of specific odors. Restrained bees were trained to form an odor-specific LTM in an appetitive Pavlovian conditioning protocol. The stability and specificity of this memory was tested behaviorally 3 $\mathrm{d}$ after conditioning. At that time, we performed both a structural and a functional analysis on a subset of 17 identified glomeruli by measuring glomerular volume under confocal microscopy, and odor-evoked activity, using in vivo calcium imaging. We show that long-term olfactory memory for a given odor is associated with volume increases in a subset of glomeruli. Independent of these structural changes, odor-evoked activity was not modified. Lastly, we show that structural glomerular plasticity can be predicted based on a putative model of interglomerular connections.
\end{abstract}

[Supplemental material is available online at http://www.learnmem.org.]

In nature, animals' survival relies on their capacity to adapt to changes in the environment by constantly learning and memorizing novel information and modifying their behavior accordingly. This capacity relies on the long-term storage of learned information involving specific stable modifications of neural networks, both in their connectivity and in the strength of synaptic transmission (Matsuzaki 2007). For such long-term memory (LTM) to be specific for particular stimuli (for instance, an odor), they must rely on specific neural traces of these stimuli in the brain. A crucial question is whether such specific traces can be tracked down to individual neural units, given the complexity of the neuronal networks usually involved in the formation of LTM. Brain regions constituted of relatively few neurons and organized in clearly identified modules offer an excellent opportunity to answer this question. Among these is the insect antennal lobe (AL), an olfactory center that shares many similarities with the vertebrate olfactory bulb, but provides numerical simplicity. In the honeybee, the AL contains $\sim 160$ interconnected neuropil subunits, the glomeruli, which can be unambiguously identified across individuals (Galizia et al. 1999a). Experiments using in vivo calcium imaging have shown that glomeruli are functional units for odorant coding and processing (Galizia et al. 1999b; $\mathrm{Ng}$ et al. 2002): Each glomerulus houses a microcircuit selectively tuned to a range of odorants, so that each odor induces a specific glomerular response pattern. Moreover, these microcircuits are plastic, as glomerular odor-evoked responses can change after conditioning (Faber et al. 1999; Sandoz et al. 2003; Yu et al. 2004) and glomerular size can vary after sensory experience (Sigg et al. 1997;

\footnotetext{
${ }^{2}$ These two authors contributed equally to this work.

${ }^{3}$ Corresponding author.

E-mail sandoz@cict.fr; fax 33-561-55-61-54.

Article is online at http://www.learnmem.org/cgi/doi/10.1101//m.1445609.
}

Brown et al. 2002; Devaud et al. 2003; Sachse et al. 2007). Except for a single genetically labeled glomerulus in mice (Jones et al. 2008), the unique property of glomeruli as identifiable functional modules has never been exploited to localize plasticity related to LTM in specific neural units. Do olfactory memory traces rely on functional and/or structural changes within a specific subset of the glomeruli? When a bee learns an odorant, a basic prediction would be that the glomeruli encoding that odorant would be the ones exhibiting plasticity (Menzel 1999). This simple prediction has never been tested before.

Here we studied whether associative olfactory learning would result in specific long-term structural and functional changes in the glomeruli coding for the learned odor. We used the associative conditioning of the proboscis extension response (PER), in which bees learn to associate an odor to sucrose reward. Bees were subjected to a spaced-trial training procedure that induced a highly odor-specific transcription-dependent LTM (Menzel 2001; Menzel et al. 2001). We measured the volume (three-dimensional reconstruction) and odor-response properties (in vivo calcium imaging) of a sample of 17 identified glomeruli. Reproducing the experiment with two different learned odors, we show that LTM is accompanied by a volume increase in specific glomeruli for each odor. We also show that the identity of the glomeruli exhibiting plasticity cannot be directly predicted from their response to the learned odor, but likely derive from local processing by AL networks.

\section{Results}

In two parallel experiments, we, respectively, measured possible structural and functional changes related to olfactory LTM in AL glomeruli, studying the brains of honeybees $3 \mathrm{~d}$ after conditioning with an odorant. In order to avoid possible effects of sensory experience per se, we compared two groups of bees that had 
received the same stimulations (odorant and sucrose), with the only difference being the timing of the stimulations, which allowed one group (the paired group) to learn the association, but not the other (the unpaired group), which served as a control.

\section{LTM is odor specific in paired bees}

Bees from the paired group were trained along five consecutive trials, in which an odor (1-hexanol or 1-nonanol) was paired with sucrose solution (Fig. 1, left). As shown (Fig. 1A: 1-hexanol; Fig. 1B: 1-nonanol), these bees learned the association efficiently (Cochran's $Q$-test, for both odors, $Q>96.7,4 \mathrm{df}, P<0.001$ ) and reached $85 \%-90 \%$ conditioned responses by the third trial. In contrast, bees from the unpaired group experienced noncontingent presentations of odorant and sucrose and showed lowresponse levels to both odorants. Thus, learning performances were significantly different between paired and unpaired groups (Mann-Whitney $U$-test: $U>62.5, P<0.001$ ), thereby showing the associative basis of acquisition performances.

Bees from both groups also differed in their responses when tested for their memory $3 \mathrm{~d}$ later (Fig. 1, right). Bees from the paired groups responded more to the learned odor $(56 \%-73 \%)$ than to the novel odor $(6 \%-12 \%)$. This difference was significant (for both odor groups, McNemar's test, $\chi^{2}>11.2, P<0.001$ ). In contrast, in the unpaired groups, responses to the experienced odor were very low ( $3 \%$ for both groups) and not different from responses to the novel odor $\left(3 \%-6 \%\right.$, McNemar's test, $\chi^{2}<0.5$, NS). In an additional experiment, we verified that the responses observed at $3 \mathrm{~d}$ in paired bees corresponded to bona fide (transcription dependent) late LTM (Menzel 2001; Schwärzel and Müller 2006) by treating paired bees with the transcription inhibitor Actinomycin D. As expected, this treatment abolished responses to the CS after $3 \mathrm{~d}$ (Supplemental Fig. S1). These results show that bees from the paired groups possessed a robust and specific transcription-dependent LTM for the learned odor $3 \mathrm{~d}$ after conditioning, while bees from the unpaired group had no such memory. Bees used in calcium imaging experiments and their yoked counterparts (see Materials and Methods) showed a similar pattern of responses (Supplemental Fig. S2).

\section{LTM is associated with increased volume in specific glomeruli}

We asked whether olfactory LTM as found in bees of the paired groups is accompanied by any detectable structural plasticity in the AL in comparison to bees of the unpaired groups without such memory. Based on their recognizable shape and respective position, we selected a set of 17 glomeruli (Fig. 2 , inset) that were identified and three-dimensionally reconstructed in 51 individuals, thus allowing the measurement of their volumes.

Within a given experimental group, glomerular volume varies greatly between different glomeruli (e.g., 17 and 23), but each glomerulus shows low interindividual variability within a group (Fig. 2A: 1-hexanol treatment; Fig. 2B: 1-nonanol treatment). However, glomerular volumes differed significantly between paired and unpaired groups, as shown by a three-way repeated-measure ANOVA with treatment (paired vs. unpaired), learned odor (1-hexanol vs. 1-nonanol), and glomerulus (repeated measure) as factors. Indeed, we found a significant treatment effect, revealing a significant increase of glomerulus volume in the paired group relative to the unpaired group $\left(F_{(1,47)}=5.09, P<\right.$ $0.05)$. As expected, we also found a significant glomerulus effect corresponding to obvious differences in volume between glomeruli $\left(F_{(16,752)}=188.7, P<0.001\right)$. Most importantly, we found a significant treatment $\times$ glomerulus interaction, revealing that volume increase in bees of the paired group relative to the unpaired group was not homogeneous, but affected some glomeruli more than others $\left(F_{(16,752)}=3.44, P<0.001\right)$. The factor learned odor and interactions with the other factors were not significant (learned odor: $F_{(1,47)}=0.61, \mathrm{NS}$; interactions: $F_{(16,752)}<$ 0.91 , NS). Thus, the close temporal association of odor and sucrose in the paired group induced a significant glomerulusspecific volume increase observable $3 \mathrm{~d}$ later and related to olfactory LTM.

We next checked whether this volume increase was observed for both conditioning odors when tested separately. For each odor, we thus carried out a twoway ANOVA with treatment (paired vs. unpaired) and glomerulus (repeated measure) as factors. Both analyses confirmed a strong glomerulus-specific volume increase (treatment $\times$ glomerulus interaction: $F_{(16,352)}=2.36, P<0.01$ for 1-hexanol and $F_{(16,400)}=2.06, P<0.01$ for 1-nonanol), although the overall volume increase was marginally nonsignificant (treatment effect: $F_{(1,22)}=3.81, P=0.06$ for 1-hexanol, and $F_{(1,25)}=2.52, P=0.12$ for 1-nonanol). As above, the glomerulus effect was significant $\left(F_{(16,352)}=101.7, P<\right.$ 0.001 for 1-hexanol and $F_{(16,400)}=94.1$, $P<0.001$ for 1-nonanol). Thus, a significant glomerulus-specific volume increase was found for both conditioned odors. Since the observed glomerulus volume increase was not homogeneous among glomeruli, we next evaluated the volume difference between paired and unpaired 
A

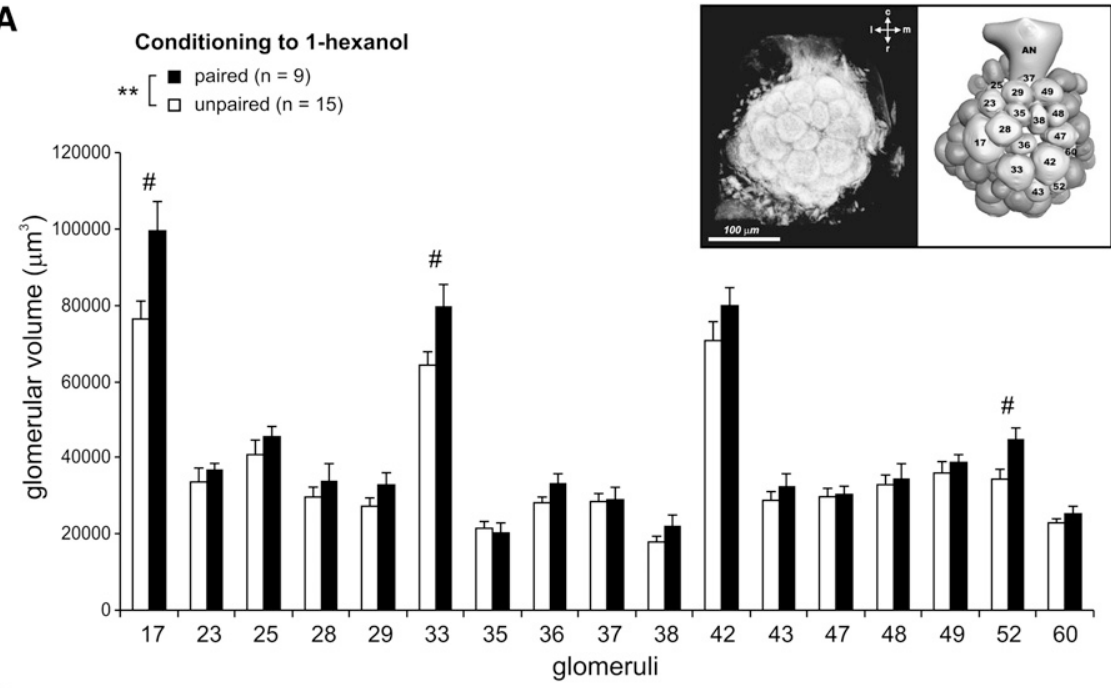

B

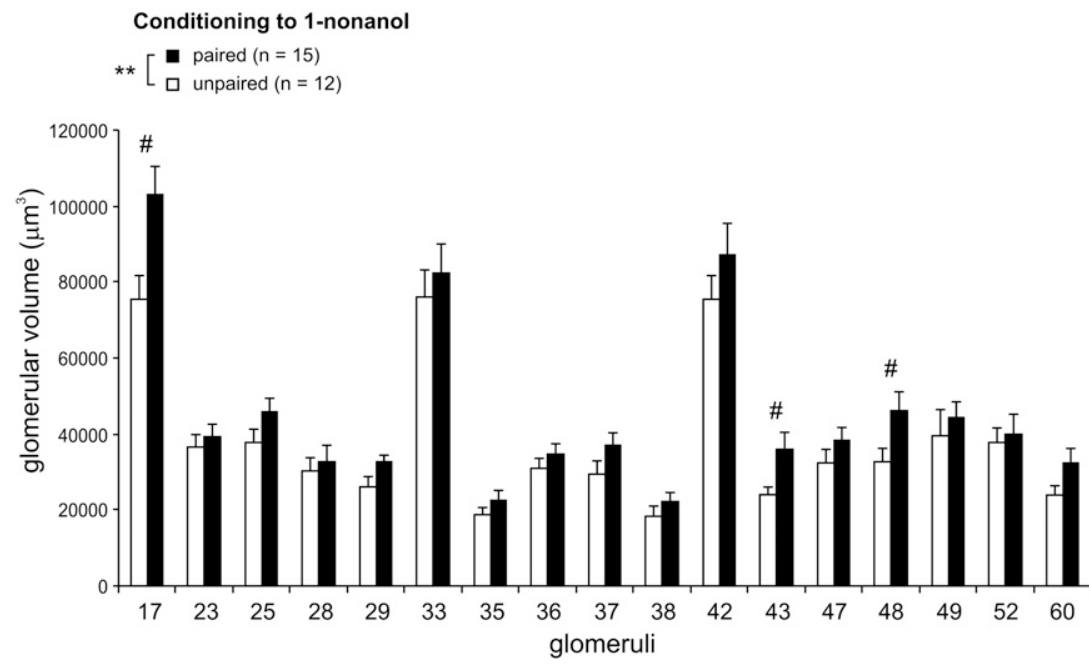

Figure 2. Olfactory LTM leads to topical increases in glomerular volume. Mean glomerular volume ( \pm SEM) is presented in 17 identified glomeruli, in paired and unpaired bees treated with either 1-hexanol $(A)$ or 1-nonanol $(B)$. The inset (top, right corner) corresponds to a three-dimensional representation of a left $\mathrm{AL}$ and the three-dimensional reconstruction of the 17 identified glomeruli. Specific glomeruli showed increased mean volume in paired animals relative to unpaired animals (ANOVA, treatment $\times$ glomerulus interaction, $F_{(16,352)}>2.06, P<0.01$ in both cases). This volume increase was observed in different glomeruli after treatment with 1-hexanol $(17,33$, and 52$)$ or with 1 -nonanol $(17,43$, and 48). (\#) $P<0.05$, post-hoc t-test.

groups for each glomerulus. For the group conditioned with 1-hexanol (Fig. 2A), three glomeruli, 17, 33, and 52, showed a volume increase above the threshold $(t>2.40,22 \mathrm{df}, P<0.05)$. For the group conditioned with 1-nonanol (Fig. 2B), also three glomeruli, 17, 43, and 48, exhibited volume increases above threshold $(t>2.09,25 \mathrm{df}, P<0.05)$. Taken together, these results show that LTM of an odor-sucrose association is accompanied by volume changes in specific subsets of glomeruli.

\section{LTM is not associated with changes in the density of odor-evoked activity}

As changes in synaptic strength are expected to be induced during memory formation, we asked whether besides inducing structural changes in the $\mathrm{AL}$, if olfactory memory was also associated with functional changes (i.e., changes in odor-evoked activity) in specific glomeruli. We used calcium imaging following bath application of Calcium Green-2AM to record neural activity of the same 17 glomeruli to six different odorants (two of which were the learned odors: 1-hexanol and 1-nonanol) in 63 individuals. To measure possible functional changes independent of volume changes, we measured odor-evoked activity on a constant surface $\left(625 \mu \mathrm{m}^{2}\right)$ for all glomeruli and all bees (see Materials and Methods). We thus examined the glomerular responses recorded to the learned odor (1-hexanol or 1-nonanol) vs. the novel odor (respectively, 1-nonanol or 1-hexanol). Overall, the two odors yielded very similar response profiles in paired and unpaired groups, independent of whether each odor was learned (Fig. 3) or novel (Supplemental Fig. S3). Consistently, a 4-way repeated-factor ANOVA with the factors treatment (paired vs. unpaired), learned odor (1-hexanol vs. 1-nonanol), glomerulus (repeated measure), and presented odor (1-hexanol vs. 1-nonanol) revealed neither an effect of treatment $\left(F_{(1,118)}=0.7, \mathrm{NS}\right)$ nor a significant interaction between treatment and other factors $(F<2.9$, NS in all cases). Three-way ANOVAs performed for each odor separately also yielded nonsignificant results $(F<0.87$ NS in all cases).

We then evaluated whether subtle changes in glomerular response patterns, which might not be detected by the ANOVA, could make the learned odor more easily discriminable from other odorants after conditioning. To test this hypothesis, we calculated Euclidian distances between activity patterns to 1-hexanol and 1-nonanol, a common way to quantify similarity between glomerular representations (e.g., Sandoz et al. 2003). We hypothesized that the neural representations of these two odors could become more different as a result of conditioning, thus leading to larger distances between them in the paired than in the unpaired group. However, this was not the case (Supplemental Fig. S4; 1-hexanol treatment: $t=0.07$, $29 \mathrm{df}$, NS; 1-nonanol treatment: $t=0.36,30 \mathrm{df}$, NS). Moreover, paired and unpaired groups did not differ regarding Euclidian distances between the learned odor and any other odor tested (data not shown).

Finally, we asked whether LTM might induce a change in the overall responsiveness of the glomeruli, affecting responses to all odorants, including those not experienced previously by the animal. We estimated such "glomerular responsiveness" by summing for each glomerulus the responses obtained to the whole panel of six tested odorants. Here again, we found no difference between paired and unpaired groups (Supplemental Fig. S5). We conclude that when recorded on a constant glomerular surface, LTM is not accompanied by any significant change in odorantinduced calcium responses. However, because the volume of specific glomeruli increased in paired groups, overall glomerular activity may indeed increase in proportion to this volume increase. 
A

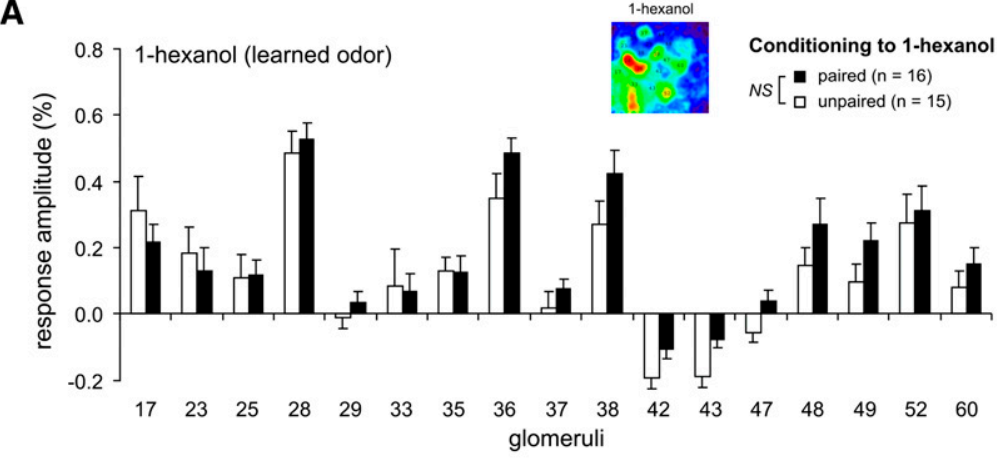

B

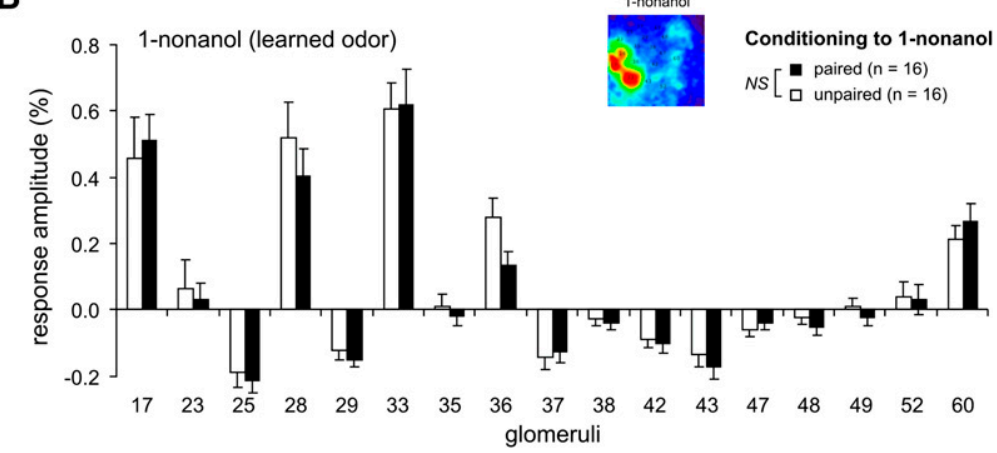

Figure 3. Olfactory LTM does not modify the density of glomerular calcium responses to the learned odor. Signal density is measured on a constant surface in each glomerulus $\left(625 \mu \mathrm{m}^{2}\right)$ after bath application of Calcium Green 2-AM. Glomerular response profiles observed to the learned odor, 1-hexanol $(A)$ or 1-nonanol $(B)$ are plotted as mean signal amplitude $(\Delta \mathrm{F} / \mathrm{F}(\%) \pm \mathrm{SEM})$. Overall, a given odor yielded very similar response profiles in paired and unpaired groups (ANOVA, treatment $\times$ glomerulus interaction: $F_{(16,464)}=0.87$, NS for 1 -hexanol; $F_{(16,480)}=0.52$, NS for 1 -nonanol). (NS) Not significant.

\section{Glomerular plasticity is predicted by a putative model of interglomerular inhibition}

Since a significant and reproducible volume increase was measured in different subsets of glomeruli in individuals with an odorspecific LTM, a simple hypothesis was that these subsets corresponded to the glomeruli activated by the conditioned stimulus (CS) during conditioning. Indeed, such glomeruli should receive concomitant inputs corresponding to the odor and to the sucrose reward during conditioning (Menzel 1999). We tested this prediction by comparing the maps of relative volume increase after conditioning to these odors (percent volume difference within each glomerulus between paired and unpaired bees; Fig. 4A) with "naive" odor-response patterns to 1-hexanol and 1-nonanol (taken from unpaired individuals that did not form any memory; Fig. 4B). A visual comparison clearly shows that though some glomeruli responding to the odorant indeed increased in volume after conditioning (e.g., glomeruli 28 and 38 in the 1-hexanol group), this is not the most frequent case. On the one hand, some glomeruli responded strongly to the odorant but did not undergo any volume change (e.g., glomerulus 33 in the 1-nonanol group). On the other hand, some glomeruli showed little or no response to the odorant but still changed markedly in volume after conditioning (e.g., glomerulus 48 in the 1-nonanol group). This was confirmed by the absence of any significant correlation between the change in glomerulus volume and the amplitude of the calcium response to the conditioned odorant (Fig. $4 \mathrm{~B} ; r^{2}=$ $0.0002,30 \mathrm{df}, \mathrm{NS})$. We conclude that the increase in volume takes place in a subset of glomeruli different from those activated by the learned odor during conditioning.
We then reasoned that plasticity may be the product of neural processing by the AL network. In the bee, networks of inhibitory interneurons are known to interconnect the glomeruli and to shape odor representation (Fonta et al. 1993; Sachse and Galizia 2002). Previous work comparing the result of computational modeling and imaging experiments established that the transformation of odor representation between $\mathrm{AL}$ input and output is best achieved by an interglomerular inhibition based on functional similarity between glomeruli (Linster et al. 2005). The investigators thus established a matrix of interglomerular inhibition from cross-correlation of the responses of the glomeruli to a range of 60 odorants (Table I in Linster et al. 2005). According to this matrix, we thus calculated for each of the 16 glomeruli in common with our study how much inhibition this glomerulus would get from the 15 other glomeruli when the CS was presented (see Materials and Methods for this calculation), and represented maps of such CS-induced inhibition (Fig. 4C). When representing volume changes associated with LTM as a function of CSinduced inhibition, we found a significant negative correlation (Fig. 4C; $r^{2}=$ $0.216,30 \mathrm{df}, P=0.007)$. Thus, the glomeruli that were the least inhibited when the CS was presented showed the most important volume increase $3 \mathrm{~d}$ after conditioning. We conclude that LTM-associated glomerular plasticity may depend on the balance between inhibitory and excitatory inputs received by a given glomeruli from the whole AL network.

\section{Discussion}

Using appetitive olfactory conditioning of the PER, we provide a simultaneous assessment of structural and functional plasticity underlying long-term olfactory memory formation in a welldefined neural network. This combined analysis, performed for the first time, shows that when bees remember an odor-sucrose association over days, they form a transcription-dependent memory that is highly specific for the conditioned odor, which is paralleled by clear and localized structural modifications in the primary olfactory center, the AL. Thus, in the honeybee, LTM formation (Menzel 2001; Schwärzel and Müller 2006) is accompanied by selective volume increases of individual glomeruli, without change in the density of odor-evoked calcium signals.

\section{Changes in glomerular volume} as a possible memory trace

Olfactory glomeruli are plastic structures undergoing volume changes even during adulthood, depending on previous experience (Drosophila: Devaud et al. [2001, 2003]; Sachse et al. [2007]; Apis: Winnington et al. [1996]; Sigg et al. [1997]). The latter studies showed significant volume changes in glomeruli after foraging experience, although the bees' actual activity during foraging was not controlled. Thus, changes could be due to different factors: intensive olfactory processing, associative olfactory learning of floral cues, non-olfactory effects, or a combination of these. To overcome 
A

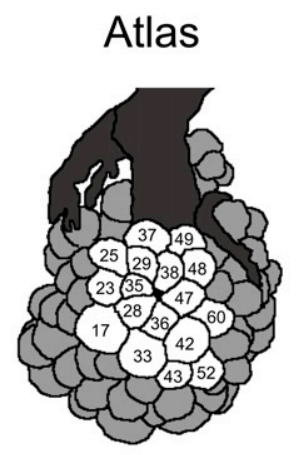

B

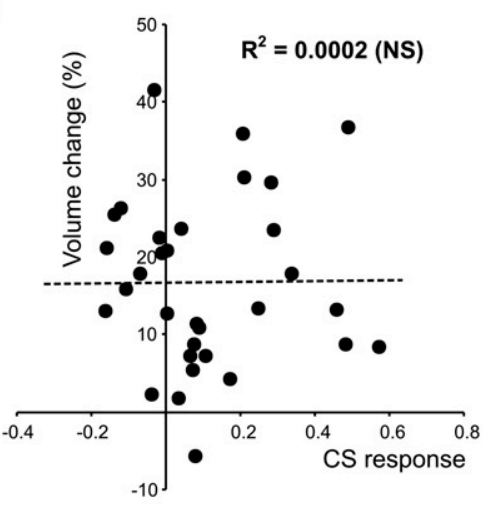

C

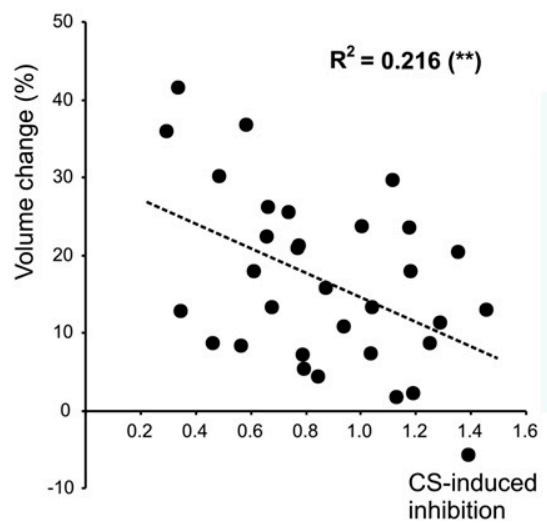

Figure 4. Glomerular structural plasticity is predicted by a putative model of interglomerular inhibition. (A) From left to right: anatomical map of the 17 studied glomeruli (Atlas); maps of glomerular volume change (as percent of volume in unpaired bees; $100 \%=$ maximal relative variation) $(B$, left) Glomerulus-wise correlation between $C S$ response, as measured in bees from the unpaired group $(\Delta \mathrm{F} / \mathrm{F} \%, n=31)$ and volume change (percent volume increase relative to unpaired bees). No relationship appeared between the two variables $\left(\mathrm{R}^{2}=0.0002, \mathrm{NS}\right)$. (Right) Maps of glomerular activity for the two learned odors (percent of maximal recorded activity, $n=31$ ). ( $C$, left) Glomerulus-wise correlation between CS-induced inhibition, calculated from the response pattern to the CS and the weights of inhibition between the glomeruli (Linster et al. 2005) and volume change (percent volume increase relative to unpaired bees). A significant negative correlation was found between the two variables $\left(R^{2}=0.216, P=0.007\right)$. (Right) Maps of CS-induced inhibition for the two learned odors (percent of maximal inhibition). Thus, glomerular plasticity appears in the glomeruli that are less inhibited as a result of CS presentation. (NS) Not significant; $\left({ }^{* *}\right) P<0.01$.

this limitation, we worked under controlled laboratory conditions with experimental and control animals receiving exactly the same number of olfactory stimulations and sucrose reinforcements, differing only in the temporal association of both stimuli. After $3 \mathrm{~d}$, pairing of odor and sucrose specifically led to volume increases in a subset of glomeruli. It could be argued that explicitly unpairing odor and sucrose might lead to LTM of a CS-no US association (Sandoz and Pham-Delègue 2004), in a way akin to latent inhibition (Abramson and Bitterman 1986; Chandra et al. 2000), and that the neural changes observed here may also include plasticity due to the CS-no US association. We thus tested this possibility in a control experiment (Supplemental material; Supplemental Fig. S6). We found that $3 \mathrm{~d}$ after our unpaired training, bees could learn the experienced odor in a conditioning procedure as well as naïve bees or bees subjected to odor-only or sucrose-only presentations (Supplemental Fig. S6). Thus, no memory of the unpaired training was present at long term. Therefore, comparing paired and unpaired bees allowed evaluating plasticity linked to odor-sucrose LTM in paired bees. After $3 \mathrm{~d}$, pairing of odor and sucrose specifically led to volume increases in a subset of glomeruli. Hence, part of the glomerular structural changes previously reported after a foraging experience could be due to learning of specific rewarded odor cues.

Which mechanisms underlie such plasticity? Experience-dependent changes in glomerular volume have been correlated with variations in synapse number in insects (Devaud et al. 2001; Brown et al. 2004), although this may not be the only process involved (Brown et al. 2002). However, other processes may be at work, since glomerular volume and synapse counts are not always correlated (Brown et al. 2002). For example, glomeruli are wrapped by glial processes that play a crucial role during their development (e.g., Boeckh and Tolbert 1993), and thus might contribute to the volume changes observed here. Indeed, astrocytic contributions to memory consolidation have been reported in vertebrates (e.g. Gibbs et al. 2008). However, at this point we cannot ascribe the memory trace to a specific cell population or process; we favor the former hypothesis of an increase in synapse numbers, as control experiments showed a good correlation between patterns of intraglomerular staining obtained with neutral red and with a synaptic marker, antisynapsin (Klagges et al. 1996; Supplemental Fig. S7). In addition, signaling pathways known to underlie synaptic plasticity are triggered in the AL during LTM formation (Grünbaum and Müller 1998; Müller 2000), and may serve as molecular bases for synaptic rearrangements in the glomeruli. However, at this point we can only speculate on the processes underlying the observed volume changes, although our results are compatible with the idea that connectivity may be modified in the bee AL. In any case, this study confirms the importance of this brain center for associative LTM formation (Grünbaum and Müller 1998; Müller 2000; Schwärzel 
and Müller 2006). It is also important to note that our results should not imply that the ALs are the only brain structures in which a memory trace of the odor-sucrose association is stored. Indeed, the mushroom bodies are a likely place for memory formation and storage (discussed in Menzel 2001), and may thus house part of the LTM trace. We are currently performing experiments to examine this possibility.

\section{Unchanged glomerular calcium responses to odorants in bees exhibiting LTM}

In parallel with volume measurements, we performed calcium imaging of glomerular odor-induced responses $3 \mathrm{~d}$ after conditioning. To our knowledge this is the first time functional recordings of $\mathrm{AL}$ responses were made so late after learning. Glomerular responses to odors did not differ between paired and unpaired animals when measuring calcium signals on a constant glomerular surface. This suggests that the density of neuronal activity was unchanged, but does not imply that overall activity in a glomerulus did not change with LTM formation, since those with an increased volume may increase in proportion to their overall activity. Future work using multiphotonic microscopy may allow monitoring odor-evoked activity on the whole three-dimensional volume of a glomerulus.

We used a bath application of Calcium-Green 2AM, a technique thought to predominantly reflect the activity of olfactory receptor neurons (ORNs) (Galizia and Vetter 2005). Our results thus suggest that ORNs do not change their responses upon LTM formation, but leave open the possibility that other neurons, such as projection neurons (PNs) and local interneurons (LINs), undergo long-term functional plasticity associated with memory formation (see below). This result seems at odds with data from previous works in Apis, showing plasticity of calcium responses using the same technique (Faber et al. 1999; Sandoz et al. 2003). However, these works differ from ours by important aspects. Faber et al. (1999) found increased calcium signals in response to the rewarded odorant 10-30 min after differential conditioning, with one rewarded and one nonrewarded odor. We have recently demonstrated that an intracellular calcium increase at the time of conditioning is both necessary and sufficient for inducing transcriptiondependent LTM (Perisse et al. 2009). How this calcium increase takes place in the brain and how long it lasts is still unknown, but the increased calcium response observed by Faber et al. (1999) could correspond to this process. If it reflects the calcium involved in triggering molecular cascades leading to LTM, it should have been terminated at the time of our recordings. Sandoz et al. (2003) used a bilateral differential olfactory conditioning protocol in which bees had to learn to respond differently to two odorants when presented on one or the other antenna. This led to a decorrelation of odor-evoked activity patterns between brain sides $24 \mathrm{~h}$ after conditioning. Although we do not know whether such activity changes would have persisted $2 \mathrm{~d}$ later, they may be triggered specifically for learning this complex side-specific task.

Our finding is consistent with studies in Drosophila, in which ORN activity remained unchanged after differential conditioning (Yu et al. 2004) or passive odor exposure (Sachse et al. 2007). In contrast, both studies found functional changes in PNs. Similarly, using electrophysiological recordings in locusts, Stopfer and Laurent (1999) observed changes in PN responses over repeated presentations of the same odorant. In honeybees, only one study so far has evaluated possible learning-induced plasticity in the activity of a subset of PNs, and found no significant changes within 15 min after conditioning (Peele et al. 2006). Data about longer delays are yet lacking, but could be obtained by selectively recording PN activity $3 \mathrm{~d}$ after learning.

\section{Odor specificity of the memory trace}

The AL is ideally suited for searching stimulus-specific memory traces, although in bees other brain centers are involved in their storage (Menzel 2001; Giurfa 2003, 2007; Devaud et al. 2007). Insect glomeruli, unlike their counterparts in vertebrates, can be readily identified across individuals. Since our behavioral observations show that LTM is specific for the CS, we expected ALs of paired and unpaired bees to differ only for a few specific glomeruli. Indeed, only a limited proportion of glomeruli (three out of 17) showed an increased volume. This suggests that part of the olfactory LTM is indeed stored in a subpopulation of AL synapses. Moreover, using two different learned odors, we have shown that structural changes related to distinct odorant-specific LTM appear to be restricted to different subsets of glomeruli. This fits with data from Drosophila where nonassociative olfactory memory induces odorant-selective volume changes in glomeruli (Devaud et al. 2001, 2003; Sachse et al. 2007), and confirms the idea that odorspecific memory traces can coexist in distinct subcircuits within a same network.

Such glomerular specificity could be explained by the fact that the glomeruli responding to the learned odor would be those that are most strongly activated during conditioning. This is because the bee glomeruli are primary sites of convergence between the two pathways providing information about the CS and the unconditioned stimulus (US) (Hammer 1993, 1997; Menzel 2001). Thus, CS-US pairing would induce concomitant activation from ORNs responding to the odor and from the pathway signaling sucrose reinforcement, triggering Hebbian plasticity mostly in the glomeruli activated by the learned odor. However, we found no correlation between glomerular volume changes and CS-induced activity, which allows discarding such a simple model. Rather, considering a putative matrix of interglomerular inhibition allowed making a good prediction of which glomeruli would show plasticity. Thus, long-term structural rearrangements in the AL cannot be deduced simply from CS-induced glomerular activity patterns and rather seem to obey more complex rules. This may also hold true for Drosophila, as synaptic protein synthesis induced during LTM formation was found in glomeruli that did not always respond to the odorants in naïve animals (Ashraf et al. 2006). Our observations support an alternative model that shall be used as a working framework for future studies. In this model, inhibition between glomeruli during CS presentations would hinder plasticity, so that the glomeruli that are inhibited the least would show the strongest volume increases. The inhibitory network proposed by Sachse and Galizia (2002), which consists of inhibitory LINs with a heterogeneous glomerular distribution of synaptic weights (Fonta et al. 1993), could introduce nonlinear changes that would decorrelate activity and plasticity maps (Sandoz et al. 2003). Thus, glomeruli may be affected during consolidation even if their direct olfactory inputs (ORNs) do not respond to the odorant. Interestingly, LIN networks in the fly AL have been proposed as sites of experience-dependent changes ( $\mathrm{Ng}$ et al. 2002), including glomerular volume increase (Sachse et al. 2007). This working model may also account for the absence of any evidence for functional plasticity in ORNs. We can thus make the prediction that if plasticity depends on the activity of LINs, it should lead to changes in the relative activity of PNs, thus resulting in decorrelated activity patterns between the CS and other odors. The fact that Peele et al. (2006) did not find plasticity in these neurons at short delays does not preclude the possibility that they might house a LTM trace. For this reason, recording calcium signals from PNs backfilled with Fura-dextran (Sachse and Galizia 2002; Peele et al. 2006) will be performed in order to detect possible functional correlates of the structural rearrangements observed here. 


\section{Materials and Methods}

\section{Animals}

Honeybees (Apis mellifera ligustica) were caught at the hive entrance, cooled, and restrained in harnesses that allow free movement of antennae and mouthparts (Bitterman et al. 1983). Then, the bees were fed with a droplet of $50 \% \mathrm{w} / \mathrm{w}$ sucrose solution and maintained for $3 \mathrm{~h}$ in the dark in an incubator $\left(30^{\circ} \mathrm{C}, \sim 75 \%\right.$ humidity) (Memmert).

\section{Behavioral experiments}

We designed a protocol aimed to compare animals differing in the presence (paired groups) or absence (unpaired groups) of a specific olfactory LTM, while equalizing sensory experience between groups. In paired groups, bees were subjected to an absolute PER conditioning protocol known to induce strong LTM (five trials, intertrial interval of $10 \mathrm{~min}$ ) (Menzel et al. 2001). At each trial, the CS (an odorant) was presented for $4 \mathrm{sec}$ and the US $(50 \% \mathrm{w} / \mathrm{w}$ sucrose solution) for $3 \mathrm{sec}$, with an interstimulus interval of $3 \mathrm{sec}$ (1-sec overlap). The CS was either 1-hexanol ( $>99 \%$, Fluka) or 1-nonanol (98\%, Sigma-Aldrich), so that two paired groups learning different odor-sucrose associations were run in parallel. As controls, two unpaired groups received explicitly unpaired presentations of the odor (either 1-hexanol or 1-nonanol) and sucrose (stimulations were presented 5 min apart: five odor-only and five sucrose-only presentations in a pseudorandomized sequence, 10 trials in total). Paired animals were also placed five times in the setup without any stimulation to equalize experience with the experimental context.

After training, bees were fed to satiation every evening with $50 \%$ sucrose and kept at $30^{\circ} \mathrm{C}$ in an incubator until retrieval tests. Assessment of LTM was conducted $3 \mathrm{~d}$ after training: Bees underwent unrewarded presentations of each of the two odorants (one already experienced during training, the other being novel). After the odor tests, all bees were tested for an intact PER by stimulating their antennae with sucrose. Bees showing no response were discarded from the study.

\section{Glomerular volume}

After testing for LTM, bees from the paired group responding specifically to the CS but not to the novel odorant, and bees from the unpaired group not responding to the two odorants were immediately sacrificed and their brains processed as follows.

\section{Brain preparation}

The head capsule of each bee was opened; glands and trachea covering the brain were removed. Then, brains were treated with a protease solution (from Bacillus licheniformis in propylene glycol; Sigma-Aldrich) for $20 \mathrm{~min}$, rinsed in bee saline ( $\mathrm{NaCl} 130 \mathrm{mM}, \mathrm{KCl}$ $6 \mathrm{mM}, \mathrm{MgCl}_{2} 4 \mathrm{mM}, \mathrm{CaCl}_{2} 5 \mathrm{mM}$, HEPES $10 \mathrm{mM}$, glucose $25 \mathrm{mM}$, sucrose $160 \mathrm{mM}$ ), stained $20 \mathrm{~min}$ in $4 \%$ neutral red (Michrome ${ }^{n o} 226$, Edward Gurr, Ltd.) and fixed overnight at $4^{\circ} \mathrm{C}$ in paraformaldehyde (Sigma-Aldrich, 4\% in PBS). Brains were then dissected out and dehydrated in rising concentrations of ethanol, then clarified in methyl salicylate (Sigma-Aldrich) for at least $4 \mathrm{~d}$ (at $-20^{\circ} \mathrm{C}$ ) until observation.

\section{Confocal microscopy}

Whole mounts were viewed with a confocal laser-scanning microscope (TCS2, Leica), with a $40 \times$ oil-immersion objective (Leica HPX PL apochromate, N.A. 1.25). Preparations were excited with a $\mathrm{He} / \mathrm{Ne}$ laser at $543 \mathrm{~nm}$ and fluorescence was detected between 555 and $650 \mathrm{~nm}$. Within each bee, the rostro-ventral part of each AL (Strausfeld 2002) was scanned at intervals of $2 \mu \mathrm{m}$ with $4 \times$ frame average. Complete stacks of optical sections $(512 \times 512$ pixels) were then imported in Amira 3.1 (Mercury Computer Systems). Glomeruli were identified (Galizia et al. 1999a) and reconstructed by manually tracing their contours on each section. The software provided a volume estimate of each glomerulus obtained from the drawn serial surfaces. The whole procedure was conducted blindfolded with respect to treatment.

\section{Glomerular activity}

Bees taken for functional imaging were not tested previously for their memory to avoid possible extinction before the imaging session. However, each bee subjected to imaging was yoked with another bee from the same group whose memory was assessed as described above. Thus, glomerular activity was recorded in response to the very first odor presentations after $3 \mathrm{~d}$, and the mean level of memory of these individuals was estimated from the behavioral responses of their yoked counterparts.

\section{Bee preparation}

Preparation and imaging were carried out as detailed elsewhere (Deisig et al. 2006). Briefly, bees were placed in Plexiglas chambers with their head capsule open, glands and trachea removed, and the brain was bathed for $90 \mathrm{~min}$ in $20 \mu \mathrm{L}$ of calcium-sensitive dye solution (prepared from $50 \mu \mathrm{g}$ of Calcium Green 2-AM dissolved with $50 \mu \mathrm{L}$ of pluronic in DMSO [both from Molecular Probes], in $800 \mu \mathrm{L}$ of bee saline). After thorough rinsing with saline, recordings started immediately.

\section{Calcium imaging}

Optical recordings of odor-evoked activity were carried out with a T.I.L.L. Photonics imaging system, under an epifluorescence microscope (Olympus BX51WI) with a $10 \times$ water-immersion objective (Olympus, UMPlanFL NA 0.3). Images were taken using a $640 \times 480$ pixel 12-bit monochrome CCD camera (T.I.L.L. Imago). Each measurement consisted of 100 frames at a rate of five frames/sec (integration time for each frame: 50-60 msec) with $4 \times 4$ binning on chip. Monochromatic excitation light at $475 \mathrm{~nm}$ was applied using a monochromator (T.I.L.L Polychrom IV). The filter set on the microscope was composed of a 505-nm dichroic filter and a long pass 515-nm emission filter.

Odor stimuli were injected in a constant air stream $(90 \mathrm{~mL} /$ min) directed to the bee's antennae (distance: $2 \mathrm{~cm}$ ), by diverting a secondary air stream from the main airflow through an interchangeable glass pipette containing a filter paper loaded with $4 \mu \mathrm{L}$ of pure odor substance. Odor stimulations were given at the 15 th frame for $1 \mathrm{sec}$, controlled by the imaging software (T.I.L.L. Vision). Each bee was first subjected to three runs of stimulations with 1-hexanol and 1-nonanol as these two odors were, depending on the group, the learned odor or a novel odor, and then an air control. Afterward, each bee was subjected to three runs with four other odors never used in behavioral experiments (1-heptanol, 2-heptanol, 2-nonanol, and 2-nonanone, in randomized order, all from Sigma-Aldrich). All 63 bees analyzed in this work provided three complete runs of responses to the six-odor panels. The intertrial interval between stimulations was $\sim 60 \mathrm{sec}$.

\section{Mapping of glomeruli}

For imaging data, individual glomeruli were identified by overlaying the patterns of glomerular activation to the six odors in Photoshop, and comparing them with published odor activation maps (Galizia et al. 1997; Sachse et al. 1999; Deisig et al. 2006) to the glomerular architecture obtained in our volumetric measurements and to the published anatomical atlas of the AL (Galizia et al. 1999a). This allowed construction of a glomerular mask with the same set of 17 glomeruli as used for volume measurements. From this glomerular mask, the coordinates of the different glomeruli in the calcium imaging data were determined. Glomerular mapping was done blindfolded regarding the experimental groups.

\section{Signal calculation}

Calcium-imaging data were analyzed with IDL (Research Systems, Inc.) according to Deisig et al. (2006). Three steps were carried out to calculate the signals including photon (shot) noise reduction, calculation of relative fluorescence changes $(\Delta \mathrm{F} / \mathrm{F})$, and bleaching correction. The latter was done either by subtracting, in each pixel of each frame, the median value of all the pixels of that frame, or the respective values obtained from the air stimulation. Both methods allowed stabilization of the recording baseline, leaving 
signals intact, and gave the same result (see Results). All data shown in the figures correspond to the first method.

Odor-evoked signals were stereotyped biphasic signals typically obtained with bath application of Calcium Green 2-AM, with a maximum signal of $\sim 1.4 \mathrm{sec}$ after odor onset (around frame 23). For each glomerulus, the time course of relative fluorescence changes was calculated by averaging 25 pixels $(5 \times 5)$, corresponding to a $25 \times 25-\mu \mathrm{m}$ window, well within its borders. The amplitude of odor-induced responses was calculated as the mean of three frames at signal maximum (frames 22-24) minus the mean of three frames before the stimulus (frames 12-14). This value was then used in all computations. It should be noted that because odor-evoked activity was calculated on a constant surface in all glomeruli and all bees, odor-induced activity was thus measured independent of total glomerular volume (see below).

Data analysis was originally conducted separately for the three runs of odor presentations in order to look for a possible effect of odor familiarity (Stopfer and Laurent 1999). Since we obtained the same results in all three runs, all analyses presented here are based on the average of the three runs.

\section{Statistical analysis}

\section{Behavioral data}

Behavioral data were dichotomous, as bees responded or not with a proboscis extension to the presented odors. Therefore, nonparametric tests for dichotomous data were used. For each group and each stimulus, the proportion of conditioned responses (percent response) was calculated and presented in the figures. Acquisition performances along trials were assessed within each group using Cochran's Q-test. Overall performance (the sum of responses of bees to the CS during conditioning) was compared between paired and unpaired groups using the Mann-Whitney $U$-test. Retention performances at $3 \mathrm{~d}$ were analyzed within each group by comparing responses to the learned odor and to the novel odor using McNemar's tests, and were compared between groups using $\chi^{2}$ tests.

\section{Volumetric data}

Volumetric data were subjected to a three-way ANOVA, with treatment (paired vs. unpaired) and learned odor (1-hexanol vs. 1-nonanol) as between-subject factors, and glomerulus (17 measures) as the within-subject factor. Then, two-way treatment $x$ glomerulus ANOVAs were also performed to assess the effect of conditioning with each learned odor. These analyses suffice to prove glomerulus-specific learning-induced plasticity. To give an indication of which glomeruli were the most plastic, the volumes of individual glomeruli were compared between paired and unpaired groups using $t$-tests. Due to our experimental design, this implied 17 comparisons per experiment (one for each glomerulus), and thus, according to statistical standards, a significance threshold correction should be applied. However, such corrections are very conservative (for instance, with the Bonferroni correction, $\alpha / \mathrm{k}$, with $\mathrm{k}$ being the number of comparisons, $\alpha^{\prime}=0.0029$.) and no glomerulus would be considered as significant in our case, although the general treatment $\times$ glomerulus ANOVA was significant. We thus chose to represent as potentially biologically relevant, the differences below a threshold of $\alpha=0.05$ and indicated them as "\#" in Figure 2A,B.

\section{Calcium imaging data}

First, we assessed whether calcium responses to the CS and the novel odor were changed by conditioning. We thus carried out a four-way ANOVA, with treatment and learned odor as betweensubject factors, and glomerulus and presented odor (1-hexanol or 1-nonanol) as within-subject factors. Analyses for 1-hexanol and 1-nonanol were run separately using three-way ANOVA, with treatment and learned odor as between-subject factors, and glomerulus as within-subject factor. Second, to ask whether learning made the pattern of responses to the learned odor more discriminable relative to a novel odor, we calculated in each bee the Euclidian distance between 1-hexanol and 1-nonanol representa- tions in the space of glomerular activation (each dimension representing activity in one of the 17 recorded glomeruli; for details see Sandoz et al. [2003] and Deisig et al. [2006]). Euclidian distances were compared between paired and unpaired groups using $t$-tests. Lastly, we also asked whether conditioning affected the overall glomerular response to odors. We thus calculated a "glomerular responsiveness" index by summing the responses of each glomerulus to the panel of six odorants tested in calcium imaging recordings (each averaged over three presentations). A three-way ANOVA as above was performed on this data set.

\section{Predicting glomerular plasticity}

We tested whether plasticity could be predicted by the glomerular activity to the CS. We thus correlated the volume change observed in each glomerulus with the CS-induced activity $(\Delta \mathrm{F} / \mathrm{F} \%)$ observed in this glomerulus. Odor-evoked activity from unpaired bees was chosen as reference, as these bees behaved like naïve bees in a control experiment (see Supplemental material) and calcium response patterns were recorded on a high number of individuals $(n=31)$. Correlation was assessed using Pearson's $r$ and a $t$-test.

We also tested the possibility that local processing by inhibitory interneurons might shape glomerular plasticity. Recently, Linster et al. (2005) provided a matrix of interglomerular inhibition that allowed predicting the transformation of glomerular responses from AL input to output. This matrix was built from correlation values between glomerular odor responses as calculated from calcium imaging data with a panel of 60 odorants (Galizia et al. 1999b; Sachse et al. 1999). This matrix contained 16 glomeruli in common with our study (all except glomerulus 43). We calculated for each odor CS, the pattern of inhibition that would arise when this CS is presented. For each glomerulus $j$ among the $p$ glomeruli, inhibition was calculated as $I_{j}=\sum_{i=1}^{p} R_{i} * c_{i j}$, in which $R_{i}$ is the response to the $\mathrm{CS}(\Delta \mathrm{F} / \mathrm{F} \%)$ in glomerulus $i$ and $c_{i j}$ is the strength of inhibition between glomeruli $i$ and $j$. Note that in the matrix a glomerulus does not inhibit itself $\left(c_{i i}=0\right)$. Otherwise, $c_{i j} \geq 0$. CS-induced inhibition was then subjected to a correlation analysis with volume changes as above.

All statistical tests were done using STATISTICA 5.0 (StatSoft).

\section{Acknowledgments}

We thank Professor Martin Giurfa for valuable comments on the manuscript. We are grateful to Dr. K. Jørgensen and Dr. M. Stranden (Trondheim, Norway) for initial help with antennal lobe stainings and reconstruction and to Dr. A. Acebes (Madrid, Spain) for insightful discussions. This work benefited from the excellent technical help from the RIO Imaging Microscopy unit of Paul Sabatier University, and in particular B. Ronsin and A. Leru for confocal microscopy and three-dimensional volumetric reconstruction.

\section{References}

Abramson CI, Bitterman ME. 1986. Latent inhibition in honeybees. Anim Learn Behav 14: 184-189.

Ashraf SI, McLoon AL, Sclarsic SM, Kunes S. 2006. Synaptic protein synthesis associated with memory is regulated by the RISC pathway in Drosophila. Cell 124: 191-205.

Bitterman ME, Menzel R, Fietz A, Schäfer S. 1983. Classical conditioning of proboscis extension in honeybees. I Comp Psychol A 97: 107-119.

Boeckh J, Tolbert LP. 1993. Synaptic organization and development of the antennal lobe in insects. Microsc Res Tech 24: 260-280.

Brown SM, Rapper RM, Thompson CM, Mercer AR. 2002. Stereological analysis reveals striking differences in the structural plasticity of two readily identifiable glomeruli in the antennal lobes of the adult worker honeybee. J Neurosci 22: 8514-8522.

Brown SM, Napper RM, Mercer AR. 2004. Foraging experience, glomerulus volume, and synapse number: A stereological study of the honey bee antennal lobe. J Neurobiol 60: 40-50.

Chandra SB, Hosler JS, Smith BH. 2000. Heritable variation for latent inhibition and its correlation with reversal learning in honeybees (Apis mellifera). J Comp Psychol 114: 86-97. 
Deisig N, Giurfa M, Lachnit H, Sandoz JC. 2006. Neural representation of olfactory mixtures in the honeybee antennal lobe. Eur J Neurosci 24: 1161-1174.

Devaud JM, Acebes A, Ferrús A. 2001. Odor exposure causes central adaptation and morphological changes in selected olfactory glomeruli in Drosophila. J Neurosci 21: 6274-6282.

Devaud J-M, Acebes A, Ramaswami M, Ferrus A. 2003. Structural and functional changes in the olfactory pathway of adult Drosophila take place at a critical age. I Neurobiol 56: 13-23.

Devaud JM, Blunk A, Podufall A, Giurfa M, Grünewald B. 2007. Using local anaesthetics to block neuronal activity and map specific learning tasks to the mushroom bodies of an insect brain. Eur J Neurosci 26: 31933206.

Faber T, Joerges J, Menzel R. 1999. Associative learning modifies neural representations of odors in the insect brain. Nat Neurosci 2: 74-78.

Fonta C, Sun XJ, Masson C. 1993. Morphology and spatial distribution of bee antennal lobe neurons responsive to odors. Chem Senses 18: 101119.

Galizia CG, Vetter R. 2005. Optical methods for analyzing odor-evoked activity in the insect brain. In Advances in insect sensory neuroscience (ed TA Christensen) pp. 349-392. CRC, Boca Raton, FL.

Galizia CG, Joerges J, Küttner A, Faber T, Menzel R. 1997. A semi-in vivo preparation for optical recording of the insect brain. J Neurosci Methods 76: $61-69$.

Galizia CG, McIlwrath SL, Menzel R. 1999a. A digital three-dimensional atlas of the honeybee antennal lobe based on optical sections acquired by confocal microscopy. Cell Tissue Res 295: 383-394.

Galizia CG, Sachse S, Rappert A, Menzel R. 1999b. The glomerular code for odor representation is species specific in the honeybee Apis mellifera. Nat Neurosci 2: 473-478.

Gibbs ME, Hutchinson D, Hertz L. 2008. Astrocytic involvement in learning and memory consolidation. Neurosci Biobehav Rev 32: 927-944.

Giurfa M. 2003. Cognitive neuroethology: Dissecting non-elemental learning in a honeybee brain. Curr Opin Neurobiol 13: 726-735.

Giurfa M. 2007. Behavioral and neural analysis of associative learning in the honeybee: A taste from the magic well. J Comp Physiol [A] 193: 801-824.

Grünbaum L, Müller U. 1998. Induction of a specific olfactory memory leads to a long-lasting activation of protein kinase $\mathrm{C}$ in the antennal lobe of the honeybee. J Neurosci 18: 4384-4392.

Hammer M. 1993. An identified neuron mediates the unconditioned stimulus in associative olfactory learning in honeybees. Nature 366: 59-63.

Hammer M. 1997. The neural basis of associative reward learning in honeybees. Trends Neurosci 20: 245-252.

Jones SV, Choi DC, Davis M, Ressler KJ. 2008. Learning-dependent structural plasticity in the adult olfactory pathway. J Neurosci $\mathbf{2 8}$ : 13106-13111.

Klagges BR, Heimbeck G, Godeschwege TA, Hofbauer A, Pflugfelder GO. 1996. Invertebrate synapsins: A single gene codes for several isoforms in Drosophila. J Neurosci 16: 3154-3165.

Linster C, Sachse S, Galizia CG. 2005. Computational modeling suggests that response properties rather than spatial position determine connectivity between olfactory glomeruli. J Neurophysiol 93: 34103417.

Matsuzaki M. 2007. Factors critical for the plasticity of dendritic spines and memory storage. Neurosci Res 57: 1-9.
Menzel R. 1999. Memory dynamics in the honeybee. J Comp Physiol [A] 185: 323-340.

Menzel R. 2001. Searching for the memory trace in a mini-brain, the honeybee. Learn Mem 8: 53-62.

Menzel R, Manz G, Menzel R, Greggers U. 2001. Massed and spaced learning in honeybees: The role of CS, US, the intertrial interval, and the test interval. Learn Mem 8: 198-208.

Müller U. 2000. Prolonged activation of cAMP-dependent protein kinase during conditioning induces long-term memory in honeybees. Neuron 27: 159-168.

Ng M, Roorda RD, Lima SQ, Zemelman BV, Morcillo P, Miesenböck G. 2002 Transmission of olfactory information between three populations of neurons in the antennal lobe of the fly. Neuron 36: 463-474.

Peele P, Ditzen M, Menzel R, Galizia CG. 2006. Appetitive odor learning does not change olfactory coding in a subpopulation of honeybee antennal lobe neurons. J Comp Physiol [A] 192: 1083-1103.

Perisse E, Raymond-Delpech V, Neant I, Matsumoto Y, Leclerc C, Moreau M, Sandoz JC. 2009. Early calcium increase triggers the formation of olfactory long-term memory in honeybees. BMC Biol 7: 30. doi: 10.1186/1741-7007-7-30.

Sachse S, Galizia CG. 2002. Role of inhibition for temporal and spatial odor representation in olfactory output neurons: A calcium imaging study. J Neurophysiol 87: 1106-1117.

Sachse S, Rappert A, Galizia CG. 1999. The spatial representation of chemical structures in the antennal lobe of honeybees: Steps towards the olfactory code. Eur J Neurosci 11: 3970-3982.

Sachse S, Rueckert E, Keller A, Okada R, Tanaka NK, Ito K, Vosshall LB. 2007. Activity-dependent plasticity in an olfactory circuit. Neuron 56: 838850.

Sandoz JC, Pham-Delègue MH. 2004. Spontaneous recovery after extinction of the conditioned proboscis extension response in the honeybee. Learn Mem 11: 586-597.

Sandoz JC, Galizia CG, Menzel R. 2003. Side-specific olfactory conditioning leads to more specific odor representation between sides but not within sides in the honeybee antennal lobes. Neuroscience 120: 1137-1148.

Schwärzel M, Müller U. 2006. Dynamic memory networks: Dissecting molecular mechanisms underlying associative memory in the temporal domain. Cell Mol Life Sci 63: 989-998.

Sigg D, Thompson CM, Mercer AR. 1997. Activity-dependent changes to the brain and behavior of the honey bee, Apis mellifera. J Neurosci 17: 7148-7156.

Stopfer M, Laurent G. 1999. Short-term memory in olfactory network dynamics. Nature 402: 664-668.

Strausfeld NJ. 2002. Organization of the honey bee mushroom body: Representation of the calyx within the vertical and $\gamma$ lobes. J Comp Neurol 450: 4-33.

Winnington, AP, Napper, RM, Mercer, AM. 1996. Structural plasticity of identified glomeruli in the antennal lobes of the adult worker honey bee. J. Comp. Neurol 365: 479-490.

Yu D, Ponomarev A, Davis RL. 2004. Altered representation of the spatial code for odors after olfactory classical conditioning; memory trace formation by synaptic recruitment. Neuron 42: 437-449.

Received April 6, 2009; accepted in revised form August 5, 2009. 


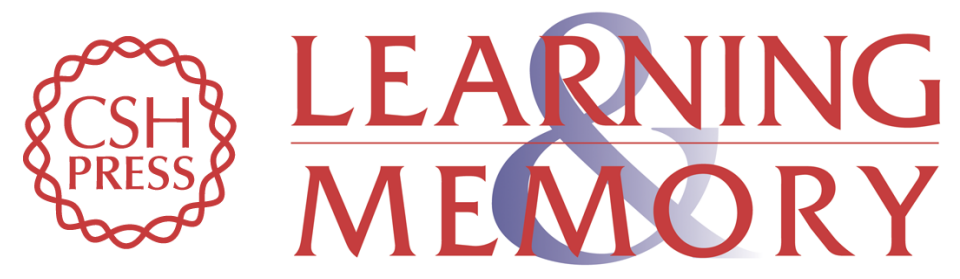

\section{Long-term memory shapes the primary olfactory center of an insect brain}

Benoît Hourcade, Emmanuel Perisse, Jean-Marc Devaud, et al.

Learn. Mem. 2009, 16:

Access the most recent version at doi:10.1101//m.1445609

\section{Supplemental http://learnmem.cshlp.org/content/suppl/2009/11/24/16.10.607.DC1 Material}

References

This article cites 44 articles, 9 of which can be accessed free at: http://learnmem.cshlp.org/content/16/10/607.full.html\#ref-list-1

\section{License}

Email Alerting

Receive free email alerts when new articles cite this article - sign up in the box at the Service top right corner of the article or click here. 